\title{
Joint line plate fixation for tibial plateau fractures caused by hyperextension varus
}

\author{
YU-CHENG HUANG, JING JIAO, WEN-JUN CHENG, FEI XIAO, WEI ZUO and JUN-WEN WANG \\ Department of Orthopaedics, Wuhan Fourth Hospital, Puai Hospital, Tongji Medical College, \\ Huazhong University of Science and Technology, Wuhan, Hubei 430032, P.R. China
}

Received May 7, 2020; Accepted October 6, 2020

DOI: $10.3892 /$ etm.2021.10053

\begin{abstract}
The present study evaluated the outcomes of internal fixation with a joint line plate in the treatment of tibial plateau fractures caused by hyperextension of the varus. The study included 25 cases (13 males and 12 females; age, 19-71 years) of tibial plateau fracture caused by hyperextension of the varus, which were treated at Puai Hospital, Tongji Medical College (Wuhan, China) between January 2015 and June 2017. Fractures were treated with internal fixations of the inner cortex with a self-clipped joint line plate made of steel. After the surgery, patients were examined immediately and at 3,6 and 12 months. Healing was evaluated by X-ray examination. All cases were cured during follow-up. After surgery, one patient developed partial necrosis of the skin margin of the incision and recovered after a dressing change. Furthermore, one patient with a concomitant peroneal nerve injury and hypoesthesia recovered after treatment with neurotrophic drugs. No screw loosening, fractures or failure of the internal fixations occurred. According to the X-ray results, there were significant differences in the tibial plateau angle (TPA) and medial posterior slope angle (m-PSA) between the pre-operative stage and 12 months post-operatively $(\mathrm{P}<0.05)$. However, no significant differences in either the TPA or m-PSA were present between the immediate post-operative stage and 12 months post-operatively $(\mathrm{P}>0.05)$. In conclusion, internal fixation with a joint line plate is an appropriate treatment for tibial plateau fractures involving the anteromedial margin with good clinical efficacy.
\end{abstract}

\section{Introduction}

Since the lower limb is not aligned with the anatomical axis of the tibia, the inner side of the tibial plateau is loaded

Correspondence to: Dr Jun-Wen Wang, Department of Orthopaedics, Wuhan Fourth Hospital, Puai Hospital, Tongji Medical College, Huazhong University of Science and Technology, 473 Hanzheng, Wuhan, Hubei 430032, P.R. China

E-mail: jingui4776663@163.com

Key words: tibial fracture, internal fracture fixation, bone plates, hyperextension, varus with $70 \%$ of the knee joint; the bone is hard and most of the fractures involved in the medial platform are large condyle cleavages or collapses (1). Tibial plateau fractures are closely related to the injury mechanism, and for its classification, Schatzker typing, Moore typing, Arbeitsgemeinschaftfür Osteosynthesefragen/Orthopaedic Trauma Association (AO/OTA) typing, three-column typing and fixation theory are most widely accepted (2-5). The correct understanding of the injury mechanism may effectively guide intra-operative reduction, enabling the accurate choice of type, location and direction of internal fixators, which is crucial for fracture prognosis (4,6,7). At present, 69\% of Schatzker IV fractures are posterior coronal fractures (8), for which the mechanism of injury is mostly caused by flexion internal force. Thus, research on typing and subtypes of Schatzker IV type fractures is intensive $(9,10)$ and there are numerous treatment methods (11-13). However, clinical reports on the typing and treatment of extended tibial plateau fractures are rare $(14,15)$. Extended tibial plateau fractures cannot be fully explained by Schatzker typing, Moore typing or AO/OTA typing (16); therefore, these fractures have been named hyperextension varus bicondylar tibial plateau (HEVBTP) fractures, the characteristics of which are as follows: Loss of posterior slope angle (PSA), a posterior cortical tension fracture, compression of the anterior bone and varus deformity. Fractures of the tibial plateau are caused by extension or hyperextension, combined with a force on the varus. The medial column is the side subjected to pressure, which means that it bears the impact of the internal condyle of the femur. When subjected to strong forces, comminuted fracture blocks of the medial and medial anterior border often occur and the articular surface may be compressed leading to its collapse; therefore, this type of fracture may involve the posterior column and even a three-column comminuted fracture. The articular surface features a backward inclination and may even turn to a forward tilt, and the medial meniscus is squeezed and may be damaged or torn. The lateral and posterolateral structure is the tension side, frequently complicating lateral collateral ligament or posterolateral complex injury and posterior cruciate ligament injury (17-20).

As a special type of fracture, tibial plateau fractures involving the anteromedial margin caused by a hyperextension varus injury in a clinical setting have rarely been reported in the literature (16). This type of fracture is usually caused by 
serious, violent injury and is characteristic of endangering the medial column, posterior column or even the three columns of the tibial plateau, which complicate the injury of the medial and lateral meniscus, posterolateral complex and posterior cruciate ligament $(14,21)$. The key to surgical treatment of tibial plateau fractures involving the anteromedial margin caused by a hyperextension varus injury is the accurate reduction of the posterior cortex and the effective support and fixation of the anterior compression bone, particularly the reduction and strong fixation of the anteromedial comminuted bone, which is a difficulty for the treatment of this type of fracture. If the treatment is improper, it inevitably results in loss of reduction, thus affecting the stability of fixation and increasing the incidence of complications. From January 2015 to June 2017, 27 patients with tibial plateau fractures caused by hyperextension varus were treated with a self-clipped joint line plate at Puai Hospital, Tongji Medical College (Wuhan, China). Among the 27 patients, 2 were lost to follow-up, and 25 had complete patient data and recovered well throughout the follow-up. In the present study, a retrospective analysis of the 25 patients is provided.

\section{Materials and methods}

Subjects. The inclusion criteria were as follows: i) Adult patients with fresh, closed fractures; ii) patients diagnosed as having a tibial plateau fracture involving the anteromedial margin caused by a hyperextension varus injury by X-ray films and CT; iii) patients with normal knee joint motion prior to injury; and iv) patients with no severe vascular or nerve injuries. Patients with open fracture, pathological fracture, severe vascular or nerve injury or complicating osteofascial compartment syndrome were excluded from the study.

A total of 25 patients were included in the present study, comprising 13 males and 12 females, aged between 19 and 71 years with a mean age of 45.2 years. All patients had closed fractures, although 2 patients had fractures with other complicated parts. The average time from injury to internal fixation was 10.7 days (range, 5 to 17 days). The causes of injury were as follows: Motor vehicle collision ( $n=6$ cases), high-fall injury (fall from over standing height, $n=5$ ), fall from an electric bicycle $(n=9)$, fall from a bicycle $(n=3)$ and fall injury (fall from standing height or less, $n=2$ ). The patients' classification based on the three-column theory was as follows (22): Simple medial column fracture ( $\mathrm{n}=4$ cases), fracture of the medial and lateral columns $(n=5)$, fracture of the medial and posterior columns $(n=9)$ and three-column fractures $(n=7)$. The classification based on AO/OTA was as follows: B2.3 ( $n=4$ cases), B3.2 $(n=9)$, C2.3 $(n=8)$ and C3.3 $(n=4)$. The basic characteristics of the patients are presented in Table SI.

Patients with simple medial column fractures were treated with a plaster cast prior to the operation and the other patients were treated with calcaneal traction. All patients received routine pre-operative swelling treatment and prevention of deep venous thrombosis of the lower extremities. Pre-operative three-dimensional CT and MRI examinations were performed to assess the extent of fracture displacement and comminution, and to assess ligament and meniscus injury. The blood circulation and sensory-motor function of the lower limbs was closely monitored prior to the operation.
The surgical incision types were as follows: Simple anteromedial incision ( $\mathrm{n}=4$ cases); simple posterior medial incision $(\mathrm{n}=7)$; anteromedial and backside inverted ' $\mathrm{L}$ ' incision $(n=2)$; anterolateral and posterior medial incision $(n=10)$; anterolateral and backside inverted ' $L$ ' incision $(n=1)$; and anterolateral and anteromedial, as well as backside inverted ' $\mathrm{L}$ ' incision $(n=1)$. All 25 patients received a bone graft during the operation, including autologous iliac bone grafts ( $n=6$ cases), allograft bone grafts $(n=17)$ and artificial bone grafts, using hydroxyapatite material $(n=2)$.

Surgical methods. Prior to the operation, proper posture selection, surgical approach and reset order were carefully planned and chosen. The supine position was used for patients who required an anteromedial approach, posterior medial approach or a conventional internal and external combined approach. The prone position was taken first after the posterior side inverted ' $\mathrm{L}$ ' incision was performed. After the internal column and the posterior column were fixed, the position was changed to a supine position. The anteromedial and/or anterolateral incision was used to reset and fix the anteromedial fracture block and the lateral column. For patients with complex, three-column fractures, the floating position may be used to observe the intra-operative reduction. After exposure, the internal and external condyle fractures were opened and the meniscus was exposed when the knee joint was turned outward. In reference to pre-operative routine MRI examination, if there was a meniscus injury, absorbable sutures were first used to repair the injury. There were 2 cases in which the medial meniscus margins had a vertical radial rupture and 4 cases in which the lateral meniscus margins had a vertical longitudinal rupture; all meniscus ruptures were repaired with absorbable sutures. During meniscus repair, it is possible to evaluate the height and shape of the tibial plateau articular surface, which is conducive to the anatomical reduction of tibial plateau fractures.

The collapsed articular surface was restored using tools of the periosteum, the top bar was placed through the fractured window and a bone allograft, autogenous bone or artificial bone graft was implanted at the collapse site. When the articular surface was satisfactorily restored, the Kirschner wire was temporarily fixed to the subchondral bone. After the fixation was confirmed by the $\mathrm{C}$-arm $\mathrm{X}$-ray machine, proper internal fixation materials were selected. The rebuilt titanium plate, $1 / 3$ tube plate, rebuilt titanium locking plate or $3.5 \mathrm{~mm}$ proximal tibia plate (PTP) titanium plate were clipped in accordance with the prebending of the medial tibia to form the joint line plate, which was then fixed to the anteromedial margin of the tibia plateau. According to the fracture conditions, combined with other internal fixation materials, such as the proximal tibial T-type locking titanium plate, the distal radius T-type titanium plate, the PTP titanium plate and the reconstruction titanium plate, final fixation was completed. For unstable bone fragments involving articular surface fixation, small hollow lag screws or absorbable sutures may be used for the fix. After the bone structure was fixed, the stability of the knee joint ligament was examined. In 2 cases in which an avulsion fracture at the insertion point of the posterior cruciate occurred, the ligaments were fixed with hollow screws after reduction. In 4 cases in which the lateral collateral ligament 
was avulsed from the fibular head, exposing the fibula capitulum through the lateral incision, wire anchors were placed and the avulsion was repaired by suture. No medial collateral ligament, anterior cruciate ligament or posterior cruciate ligament rupture were identified in any of the patients. The wound drainage tube was routinely retained after the operation.

Post-operative treatment and follow-up. All patients were treated with a cotton pad to bandage the wound to reduce bleeding after the operation. At the same time, the affected limb was raised to reduce wound and limb swelling. Low-molecular-weight heparin was administered $12 \mathrm{~h}$ after the operation to prevent deep vein thrombosis of the lower limb. After $48 \mathrm{~h}$, the wound drainage tube was removed and knee joint flexion and extension function exercises were performed under the protection of braces.

All patients underwent examination immediately after the operation and at 3,6 and 12 months after the operation. The healing time of the fracture was judged by X-ray and clinical examination, and the time of full weight-bearing walking was recorded. Any complications after the operation and during follow-up were recorded. Due to the medial tibial plateau bearing most of the load of the knee joint, if the fracture block of the anteromedial platform was seriously crushed, the time of partial weight load was postponed accordingly. It may be suggested that the patient should be supported for up to 3 months after the operation. The time-point of full-weight load-bearing was finally determined by X-ray according to the degree of fracture grinding and the healing conditions.

The tibial plateau angle (TPA) and PSA were measured by $\mathrm{X}$-ray. The TPA and PSA of the tibial plateau prior to operation, immediately after operation and 12 months after the operation were compared. Knee joint function of the affected limb was measured 12 months after the operation and was evaluated according to the knee joint Hospital for Special Surgery (HSS) standard score (23).

Statistical analysis. The statistical software SPSS 19.0 (IBM, Corp.) was used for statistical analyses. First, the Shapiro-Wilk test was used to determine whether the measurement data had a normal distribution. The tibial plateau TPA, the medial PSA (m-PSA) and the lateral PSA (1-PSA) had normal distribution data, and the variance was homogeneous; therefore, values were expressed as the mean \pm standard deviation. Comparisons of the tibial plateau TPA and PSA prior to the operation, immediately after the operation and 12 months after the operation were analyzed by repeated-measures ANOVA with further pairwise comparisons by the least-significant difference t-test. $\mathrm{P}<0.05$ was considered to indicate a statistically significant difference.

\section{Results}

Status of fracture healing after the operation. The 25 patients were followed up for an average of 17.6 months (range, 12-30 months) and it was found that all fractures had healed. $\mathrm{X}$-ray films revealed that 20 cases achieved anatomical reduction and 3 cases had articular surface collapse by $<2 \mathrm{~mm}$. The average operation time was $48 \mathrm{~min}$ (range, 25-120 min) and the average blood loss during the operation was $110 \mathrm{ml}$ (range, 15-250 ml). The mean Rasmussen score immediately after the operation was 15.6 points (range, 12-18 points). Fracture healing was achieved in all 25 patients. The average time of healing and full weight-bearing was 12.1 weeks (range, 8-14 weeks) and 15.8 weeks (range, 13-20 weeks), respectively. The HSS scores of all the patients at 12 months post-operatively averaged 87.6 (range, 68-96), yielding an excellent to good rate of $91.5 \%$. The average range of motion of the affected knee ranged from $2.3^{\circ}$ to $125.1^{\circ} 12$ months after the operation. The data of the status of fracture healing after the operation are presented in Table SI.

TPA and posterior slope angle of the tibial plateau prior to operation and after the operation. The tibial plateau TPA and m-PSA immediately as well as 12 months after the operation were significantly different from those prior to the operation $(\mathrm{P}<0.05$; Fig. 1A and $\mathrm{B})$. However, there was no significant difference in the TPA and m-PSA between the stage immediately after the operation and 12 months after the operation ( $P>0.05$; Fig. 1A and B). No significant difference was present in the pre-operative 1-PSA between the stage immediately after the operation and 12 months after the operation $(\mathrm{P}>0.05$; Fig. 1C). Partial incision necrosis occurred post-operatively in 1 case, which uneventfully healed after wound management. Typically, the fractures of a 19-year-old male with hyperextension varus of tibial plateau fractures (Schatzker V type) caused by a motor vehicle collision (Fig. 2) and a 33-year-old female with hyperextension varus of tibial plateau fractures (Schatzker V type) caused by a high-fall injury (Fig. 3) both were healed, and there was no loss of reduction at 12 months post-surgery. In addition, there were 2 cases complicated with pre-operative common peroneal nerve injury reported with a dorsal sense of numbness, which healed after 3 months of neurotrophic drug administration. No screw loosening, plate breakage or fixation failure was detected during follow-up.

\section{Discussion}

Internal fixation materials commonly used for clinical medial platform repairs, such as 3.5 or $4.5 \mathrm{~mm}$ locking titanium plates or reconstruction titanium plates cannot be fixed to the anteromedial margin and cannot form a strong support and fixation for the broken fracture block of the anteromedial cortex due to the limited coverage or support area (24-27). In particular, cases in which the anteromedial cortical bone fracture extends to the anterolateral side, resulting in the overall comminuted collapse of the tibial plateau, are lacking effective fixation, which inevitably results in inadequate elevation of the medial articular surface of the medial platform, insufficient recovery of the obliquity, loss of later reduction, failure of internal fixation, impairment of the stability of knee joint and an increase in the incidence of complications (28). Therefore, internal fixation and material selection for anteromedial cortical bone comminuted fractures are difficult and clinical reports are currently sparse. As an example, Firoozabadi et al (16) placed $3.5 \mathrm{~mm}$ medial T-locking titanium plates in the anteromedial side as the main supporting plate, but only to fix the entire medial condyle bone block. As for a comminuted fracture involving the joint margin, scholars worldwide have attempted to use various types of internal fixation. Giordano et al (29) 

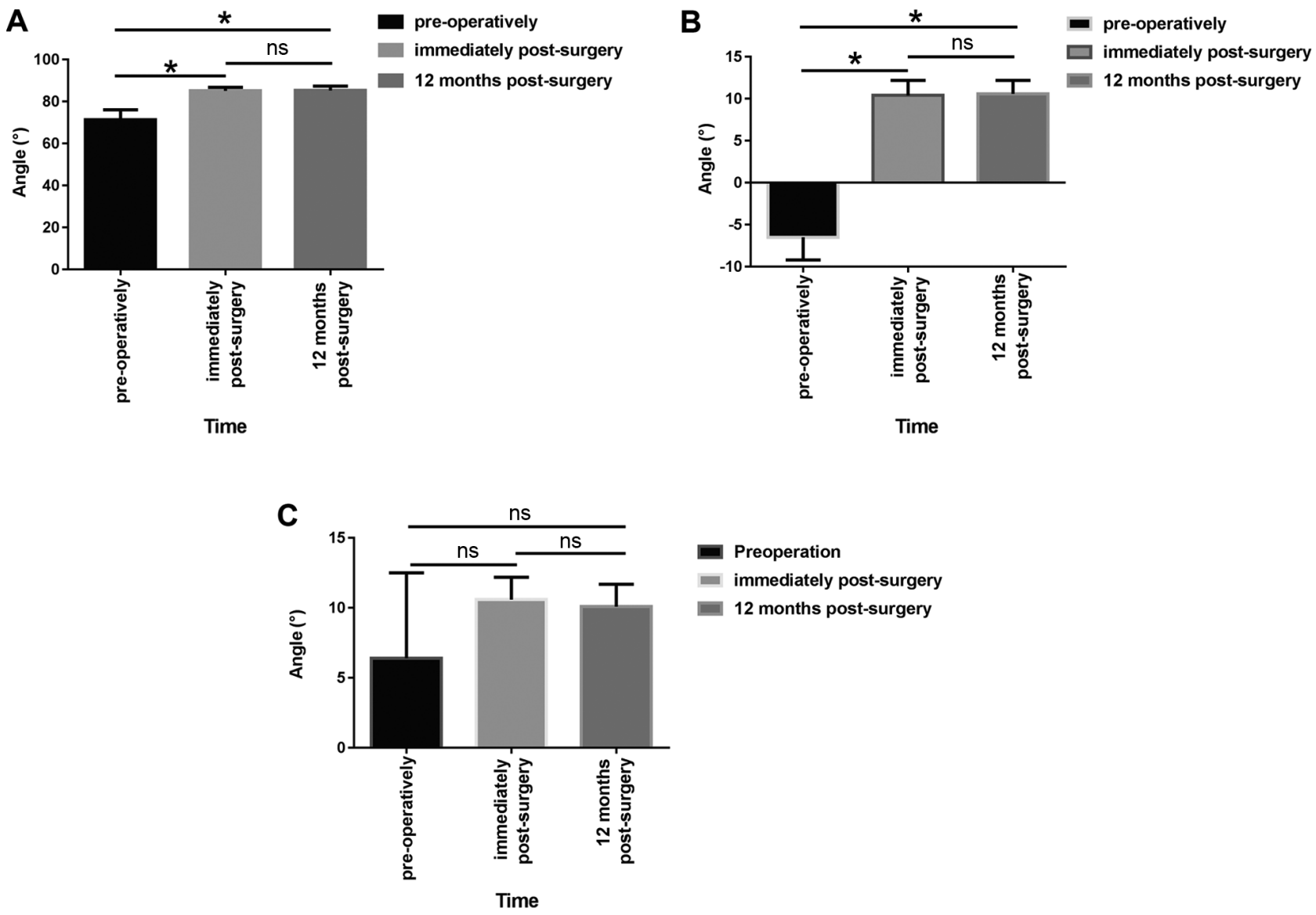

Figure 1. (A) TPA, (B) medial PSA (m-PSA) and (C) lateral PSA (1-PSA) of all patients prior to surgery, immediately after surgery and at 12 months after the operation. The TPA and m-PSA immediately after surgery were significantly different from those prior to the operation. There was no significant difference in the tibial plateau TPA or the m-PSA at 12 months after the operation vs. immediately after the operation. No significant difference in the 1-PSA was observed among the stages prior to surgery, immediately after the operation and 12 months after surgery. ${ }^{*} \mathrm{P}<0.05$. ns, no significance $(\mathrm{P}>0.05)$. TPA, tibial plateau angle; PSA, posterior slope angle.

applied a 1/3 tubular steel plate preflexion and transverse fixation to the posterior lateral joint in comminuted fractures of the posterolateral joint margin of the tibial plateau and called it 'hoop plating'. Lv et al (30) used a 2.4-mm radial distal locking plate for the treatment of a posterior lateral collapse fracture of the tibial plateau. The plate was placed closely to the articular surface, which achieved a satisfactory clinical effect. However, the 2.4 or $2.7 \mathrm{~mm}$ radial distal plate system has low strength and small coverage, is mostly used for the internal fixation of upper limb fractures and is not reported for the treatment of comminuted fractures of the medial tibial medial cortex.

In the present study, the concept of the circumference line steel plate was proposed: The shape of the internal fixation material is an arc, semi-ring or even a ring fixed to the articular cartilage along the joint edge to fix the broken fracture block. The idea is to fix the comminuted fracture blocks to the main fracture block or other stable fracture blocks of the joint surface to form the whole condyle. Through its own metaphyseal support or other metaphyseal plate bridging, the condyle was fixed to the diaphysis. At the same time, the plate was fixed to the anteromedial margin and the screw was located below the articular surface, particularly with the angle stability of the locking plate, forming 'bamboo raft fixation' technology and the cross fixation technique for the articular cartilage, which may provide a powerful mechanical support for the formation of the comminuted fracture blocks of the anteromedial margin (31).

Giordano et al (29) proposed that the 'hoop plate' may also be used for the joint edge, which may appear the same as the concept of the joint line plate of the present study, but actually it is not. The hoop plate is used to treat posterior condyle fractures of the tibial plateau, of which the $1 / 3$ tube plate is pre-bent to the posterior side of the tibial plateau to strengthen the effect of a 'buttress plate', meaning that 'hoop plates' only have a fixed function as the collar. The joint line plate is also placed on the joint edge in the present study of the anteromedial tibial plateau joint and may also be used for other parts of the fracture. At the same time, the idea of the joint line plate in fixing bone fragments of the joint edge is emphasized, similar to the 'hoop plate' or locking plate, which exerts the angle stabilizing effect of locking screws to form a 'bamboo raft fixation' effect, having a supporting function on the metaphyseal. At present, there is no such specialized type of joint line plate used for the anteromedial margin of the tibial plateau. In the present study, a self-clipped joint line plate was made based on the size and position of the fracture blocks, including the use of reconstruction titanium plates ( $n=6$ cases), $1 / 3$ tube type steel plate fixation $(n=5)$, reconstruction locking plate fixation $(\mathrm{n}=10)$ and a $3.5-\mathrm{mm}$ system PTP 
A

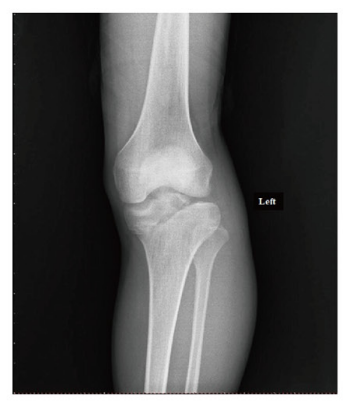

E

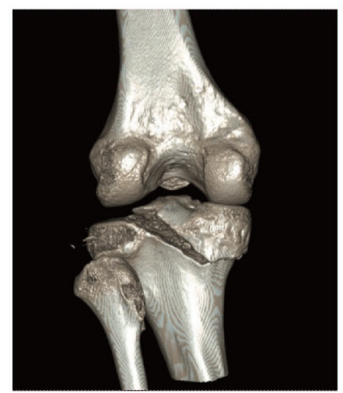

I

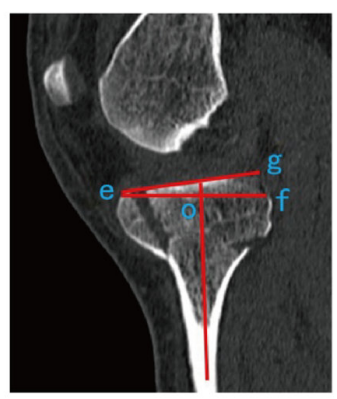

M

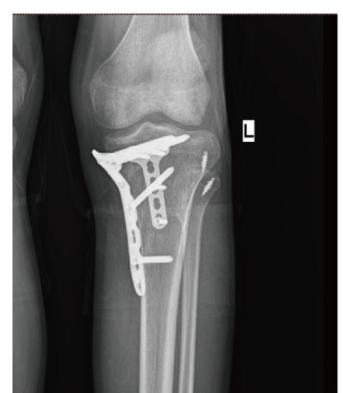

B

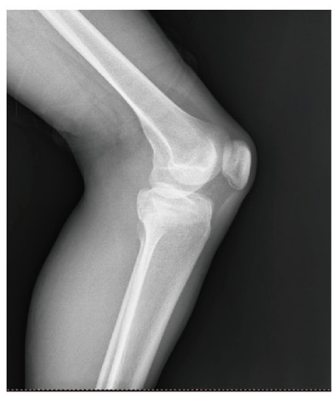

F

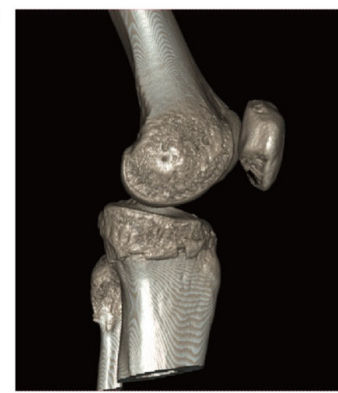

J

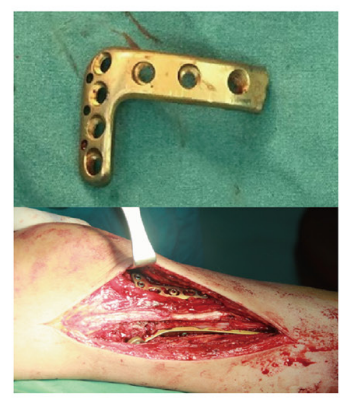

N

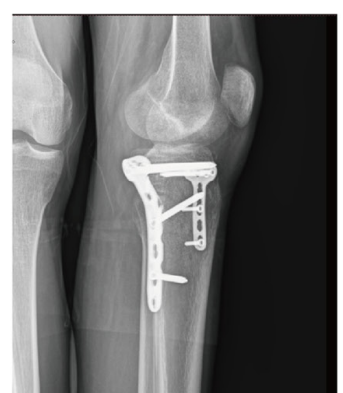

C

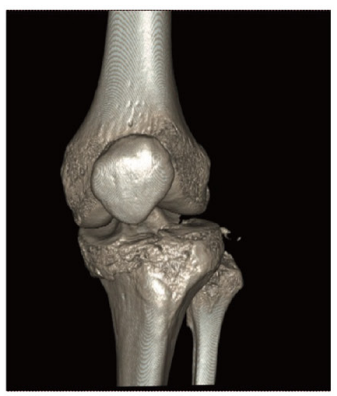

G

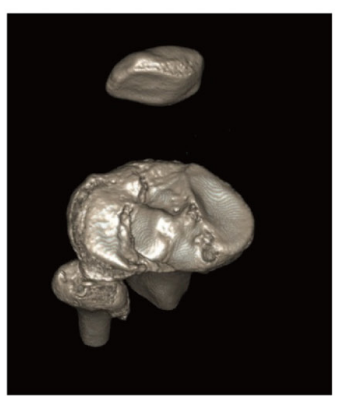

K

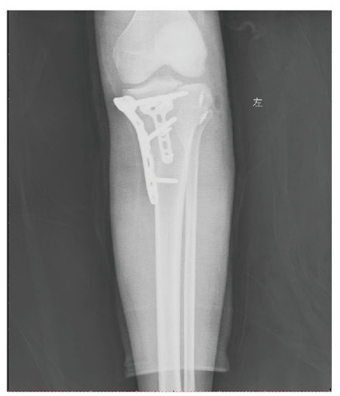

0

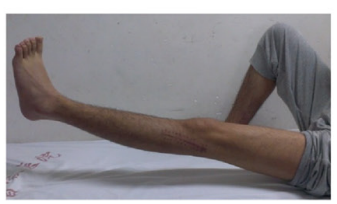

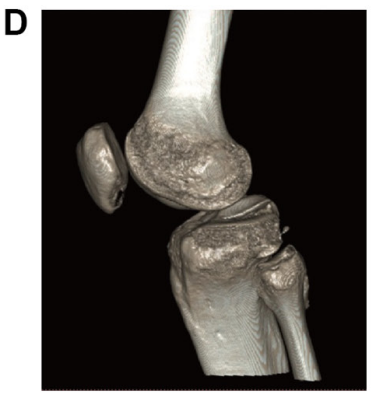

H

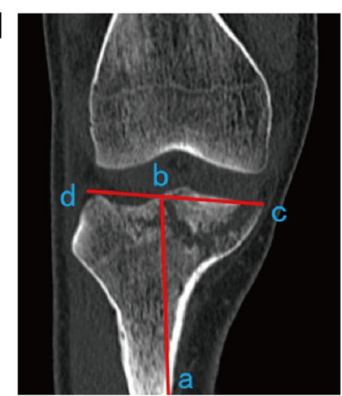

L

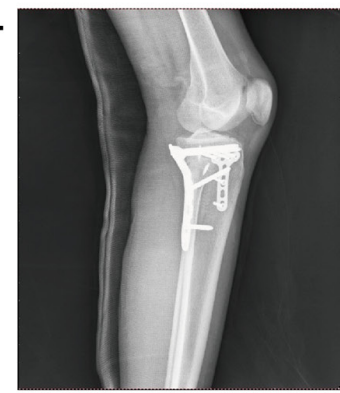

P

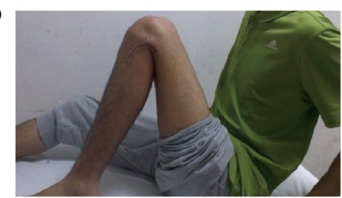

Figure 2. Representative case of a 19-year-old male with hyperextension varus of tibial plateau fractures (Schatzker V type) caused by an electric bicycle collision. Pre-operative radiographs in frontal (A) and side (B) position. Pre-operative three-dimensional CT displaying medial and lateral column fractures. (C) Anterior view. (D) Lateral view from left side. (E) Posterior view. (F) Lateral view from right side. (G) Transverse view. (H) $\angle$ abc represents the tibial plateau angle and (I) $\angle$ gef represents the posterior slope angle by CT. (J) Image of a 2.7-mm T-shaped Locking plate that was horizontally placed and its location during the operation. (K and L) Radiography was performed immediately after the operation. (M and N) Radiographs at 12 months post-surgery indicated that the fractures were healed and there was no loss of reduction. $(\mathrm{O}$ and $\mathrm{P})$ General functional position of the patient 12 months after the operation.

titanium plate fixation $(n=4)$. Among the 25 patients included in the follow-up, 20 cases achieved anatomical reduction and the tibial plateau TPA and PSA recovered satisfactorily after the operation. At one year after the operation, the tibial plateau TPA, m-PSA and 1-PSA exhibited no significant changes from the angles immediately after the surgery and the reduction was not lost. It was proven that with the fixation of the medial supporting plate, the self-clipped joint plate was able to meet the requirements of the early functional exercise of the affected limb and the recovery of the knee joint function was satisfactory after the operation.

During the operation, the height of the anteromedial platform fracture should be fully restored, along with the TPA and PSA, and may be compared with that of healthy limbs. It is crucial that the bone graft in the bone defect area is sufficient. If there are certain defects, it is suggested that an autogenous iliac bone graft may be used, with the advantages of having powerful support, effects of good bone formation, bone induction and conduction, and fastening of the fracture during healing (32). During the operation, the medial collateral ligament, the 'goose foot' and other medial stable structures should be protected to avoid post-operative instability of the knee joint (33). When prebending the joint line plate, the plate should be closely adhered to the bone to reduce irritation to the skin and soft tissue and the influence of the flexion activity of the knee joint after the operation. During the operation, if it 
A

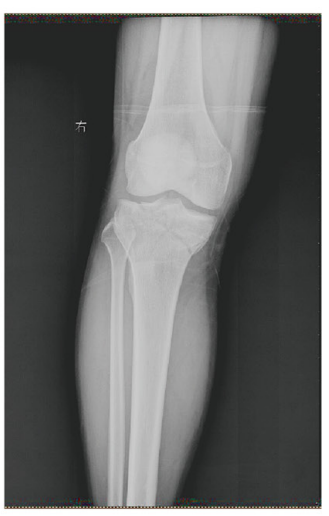

E

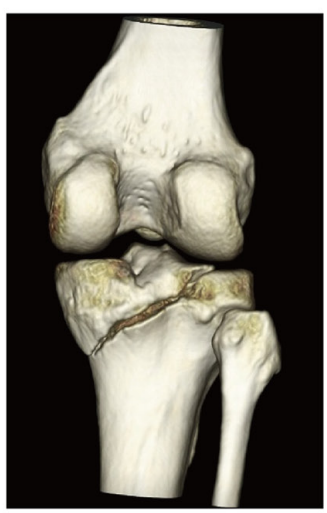

I

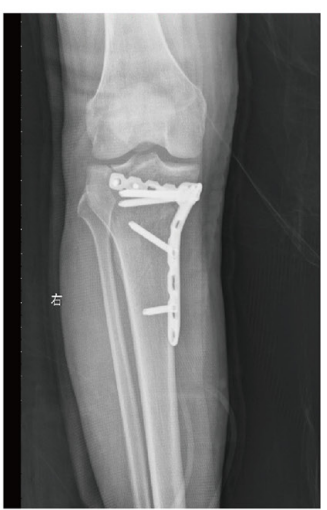

B

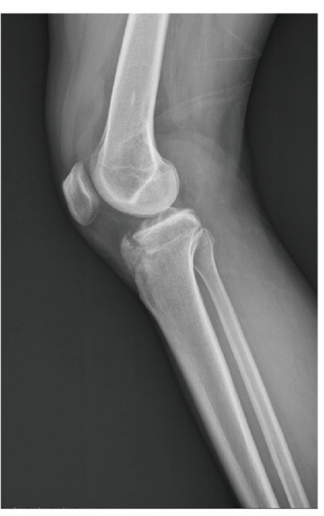

$\mathbf{F}$
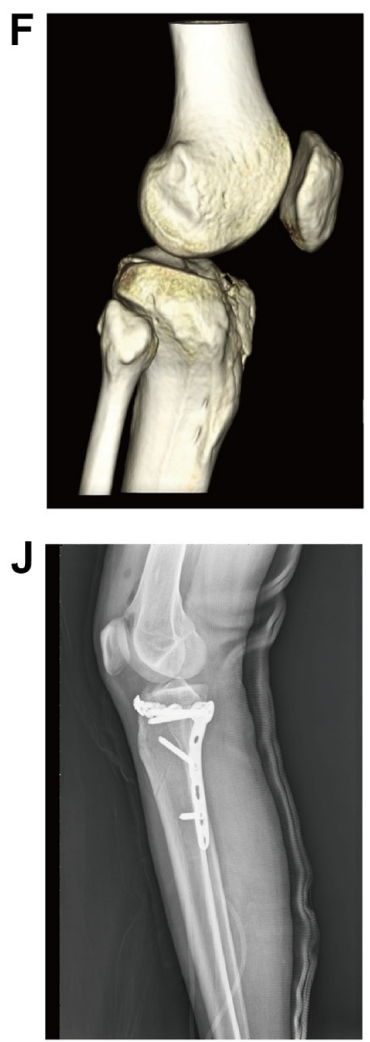

C

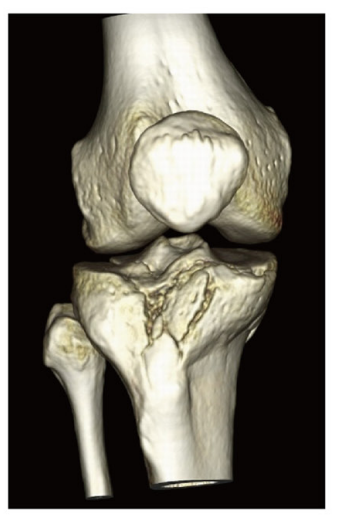

G

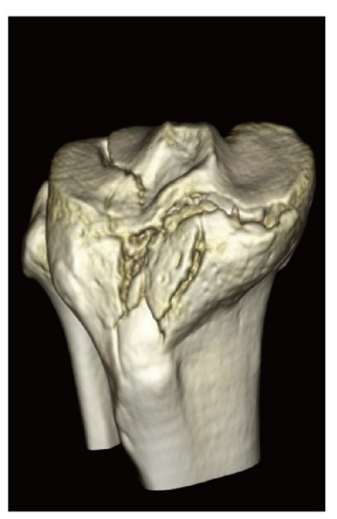

K

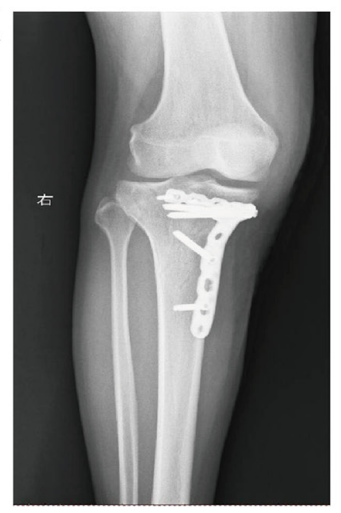

D

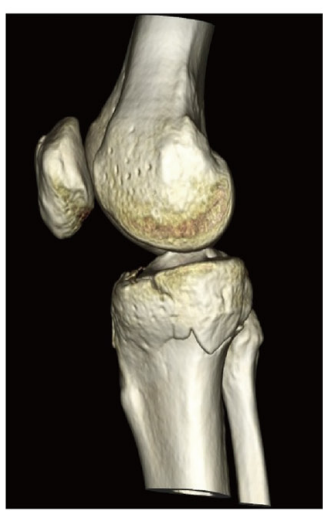

H

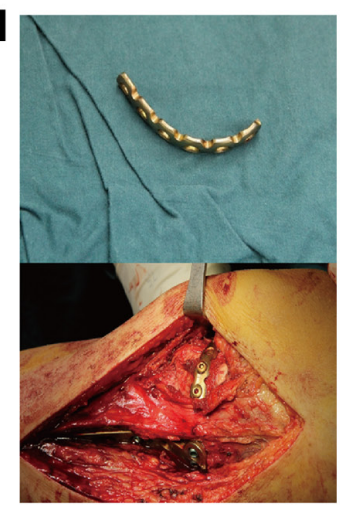

$\mathbf{L}$

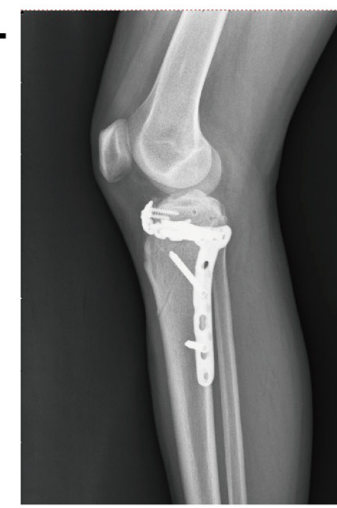

M

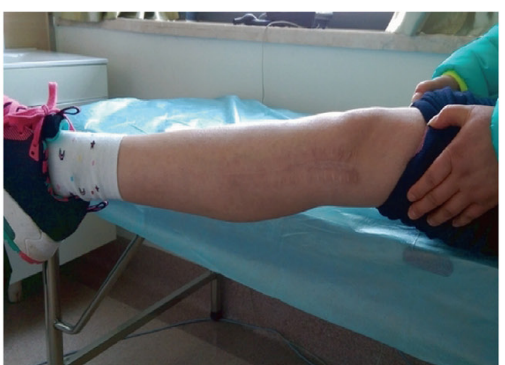

$\mathbf{N}$

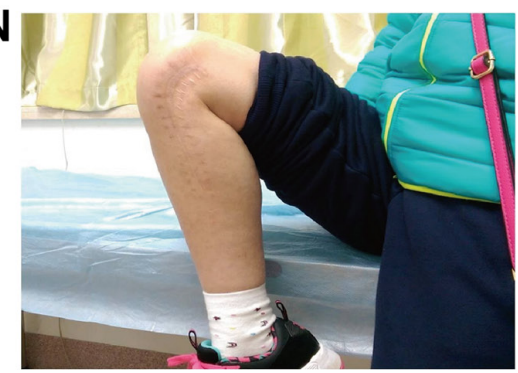

Figure 3. Representative case of a 33-year-old female with hyperextension varus of tibial plateau fractures (Schatzker V type) caused by a fall injury Pre-operative radiographs in (A) frontal and (B) side position. Pre-operative three-dimensional CT revealing three-column fractures. (C) Anterior view. (D) Lateral view from left side. (E) Posterior view. (F) Lateral view from right side. (G) Transverse view. (H) Image of a 3.5-mm rebuilt titanium plate that was horizontally placed and its location during the operation. Radiographs immediately post-surgery in (I) frontal and (J) side position. Radiographs at 12 months post-surgery in $(\mathrm{K})$ frontal and $(\mathrm{L})$ side position indicated that the fractures were healed and there was no loss of reduction. (M and $\mathrm{N}$ ) General functional position of the patient 12 months after the operation.

is necessary to reduce the dissection of the soft tissue around the incision, the use of an electric knife and clamping the edge of the skin should be avoided in order to reduce complications such as incision fat liquefaction, skin necrosis or wound infection. There may be a posterolateral complex injury in this type of fracture and particularly the lateral collateral ligament may be torn or the fibular head may have an avulsion fracture. Internal and external stress tests should be performed after the internal fixation of the intraoperative fracture reduction, the stability of the lateral collateral ligament should 
be checked and one-stage surgical repair should be performed if necessary (34).

Deficits of the present study are that the number of cases with the application of the joint line plate was limited and the follow-up time was relatively short. Further studies with more cases and further follow-up observations are required to acquire more accurate clinical treatment results. In addition, the self-clipped joint line plate has no supporting functions on the epiphysis, which is necessary to cooperate with other internal fixation materials to complete the final fixation. Therefore, according to the idea of joint line plating, it is esteemed to develop an anatomic type of joint line plating to meet clinical requirements.

\section{Acknowledgements}

Not applicable.

\section{Funding}

This study was supported by grants from the Health and Family Planning Commission of Wuhan City for Clinical Medicine Research Project (grant no. WX16D27).

\section{Availability of data and materials}

The datasets used and/or analyzed during the current study are available from the corresponding author on reasonable request.

\section{Authors' contributions}

YCH performed the experiments, analyzed the data and wrote the paper; JJ contributed to the conception of the study; WJC, FX and WZ participated in the execution of the experiment; JWW contributed to analysis and manuscript preparation. All authors read and approved the final manuscript.

\section{Ethics approval and consent to participate}

The study was approved by the ethics committee of the Wuhan Fourth Hospital, Puai Hospital, Tongji Medical College, Huazhong University of Science and Technology (Wuhan, China). All patients provided written informed consent.

\section{Patient consent for publication}

All patients agreed to publish their images and scans.

\section{Competing interests}

The authors declare that they have no competing interests.

\section{References}

1. Duan KD and Huang JR: Progress in diagnosis and treatment of posterior condylar fracture of tibial plateau. Zhongguo $\mathrm{Gu}$ Shang 32: 1173-1176, 2019 (In Chinese).

2. Lowe DT, Milone MT, Gonzalez LJ and Egol KA: Repair of tibial plateau fracture (Schatzker II). JBJS Essent Surg Tech 9: e25, 2019.

3. Kfuri M and Schatzker J: Revisiting the Schatzker classification of tibial plateau fractures. Injury 49: 2252-2263, 2018.
4. Zhang BB, Sun H, Zhan Y, He QF, Zhu Y, Wang YK and Luo CF: Reliability and repeatability of tibial plateau fracture assessment with an injury mechanism-based concept. Bone Joint Res 8: 357-366, 2019.

5. Ramponi DR and McSwigan T: Tibial plateau fractures. Adv Emerg Nurs J 40: 155-161, 2018.

6. Graham P: Tibial plateau fracture. Orthop Nurs 36: 303-305, 2017

7. Lu C, Ye G, Liu W, Wu H, Wu G and Chen J: Tibial plateau fracture related to unicompartmental knee arthroplasty: Two case reports and literature review. Medicine (Baltimore) 98: e17338, 2019.

8. Chang SM, Zhang YQ, Yao MW, Du SC, Li Q and Guo Z: Schatzker type IV medial tibial plateau fractures: A computed tomography-based morphological subclassification. Orthopedics 37: e699-e706, 2014.

9. Chang SM, Hu SJ, Zhang YQ, Yao MW, Ma Z, Wang X, Dargel J and Eysel P: A surgical protocol for bicondylar four-quadrant tibial plateau fractures. Int Orthop 38: 2559-2564, 2014.

10. Yan B, Yin W, Zhang X, Liu D, Gui K, Sun J, Chen Y and Ni M: Effectiveness analysis of surgical treatment of Schatzker type tibial plateau fractures. Zhongguo Xiu Fu Chong Jian Wai Ke Za Zhi 31: 1305-1310, 2017 (In Chinese).

11. Cherney S and Gardner MJ: Bicondylar tibial plateau fractures: Assessing and treating the medial fragment. J Knee Surg 27: 39-45, 2014.

12. He X, Ye P, Hu Y, Huang L, Zhang F, Liu G, Ruan Y and Luo C: A posterior inverted L-shaped approach for the treatment of posterior bicondylar tibial plateau fractures. Arch Orthop Trauma Surg 133: 23-28, 2013.

13. Lu KH, Lu EW, Lin CW, Yang JS and Yang SF: New insights into molecular and cellular mechanisms of zoledronate in human osteosarcoma. Pharmacol Ther 214: 107611, 2020.

14. Kołodziejczyk K, Kuliński K, Fedorowicz K, Langner M, Czubak J and Pomianowski S: Difficulties in treating complex knee injuries with fracture of posterior tibial plateau. Ortop Traumatol Rehabil 20: 293-300, 2018.

15. Yang X, Xu F, Yin Z and Wang Q: Clinical observation of $3.5 \mathrm{~mm}$ T support plate fixation for simple posterolateral tibial plateau fracture by posterolateral inverted L-shaped approach. Zhongguo Xiu Fu Chong Jian Wai Ke Za Zhi 31: 815-819, 2017 (In Chinese).

16. Firoozabadi R, Schneidkraut J, Beingessner D, Dunbar R and Barei D: Hyperextension varus bicondylar tibial plateau fracture pattern: Diagnosis and treatment strategies. J Orthop Trauma 30: e152-e157, 2016.

17. Cohen AP, King D and Gibbon AJ: Impingement fracture of the anteromedial tibial margin: A radiographic sign of combined posterolateral complex and posterior cruciate ligament disruption. Skeletal Radiol 30: 114-116, 2001.

18. Bennett DL, George MJ, El-Khoury GY, Stanley MD and Sundaram M: Anterior rim tibial plateau fractures and posterolateral corner knee injury. Emerg Radiol 10: 76-83, 2003.

19. Chiba T, Sugita T, Onuma M, Kawamata T and Umehara J: Injuries to the posterolateral aspect of the knee accompanied by compression fracture of the anterior part of the medial tibial plateau. Arthroscopy 17: 642-647, 2001.

20. Yoo JH, Kim EH, Yim SJ and Lee BI: A case of compression fracture of medial tibial plateau and medial femoral condyle combined with posterior cruciate ligament and posterolateral corner injury. Knee 16: 83-86, 2009

21. Hong F, Wang $\mathrm{N}$ and Chen GJ: Posterior medial approach inverted L-shaped incision combined with reconstruction plate for posterior condylar fracture of tibial plateau. Zhongguo $\mathrm{Gu}$ Shang 29: 1027-1032, 2016 (In Chinese).

22. Luo CF, Sun H, Zhang B and Zeng BF: Three-column fixation for complex tibial plateau fractures. J Orthop Trauma 24: 683, 2010.

23. Insall JN, Ranawat CS, Aglietti P and Shine J: A comparison of four models of total knee-replacement prostheses. J Bone Joint Surg Am 58: 754-65, 1976.

24. Dreyfuss D, Allon R, Izacson N and Hutt D: A Comparison of locking plates and intramedullary pinning for fixation of metacarpal shaft fractures. Hand (NY) 14: 27-33, 2019.

25. Wu CM, Chen YA, Liao HT, Chen CH, Pan CH and Chen CT: Surgical treatment of isolated zygomatic fracture: Outcome comparison between titanium plate and bioabsorbable plate. Asian J Surg 41: 370-376, 2018

26. Darrow BG, Weigel JP, Greenacre CB, Xie X, Liaw PK and Biskup JJ: Ex vivo biomechanical comparison of titanium locking plate, stainless steel nonlocking plate, and Tie-in external fixator applied by a dorsal approach on ostectomized humeri of pigeons (Columba livia). J Avian Med Surg 33: 29-37, 2019. 
27. Schliemann B, Seifert R, Theisen C, Gehweiler D, Wähnert D, Schulze M, Raschke MJ and Weimann A: PEEK versus titanium locking plates for proximal humerus fracture fixation: A comparative biomechanical study in two- and three-part fractures. Arch Orthop Trauma Surg 137: 63-71, 2017.

28. Maeda H, Noguchi M, Suehiro M, Mihara E and Chou Z: Analysis of surgical treatment results in patients with tibial plateau fractures. Orthoped Traumatol 42: 805-809, 2010.

29. Giordano J, Schatzker M and Kfuri M: The 'Hoop' plate for posterior bicondylar shear tibial plateau fractures: Description of a new surgical technique. J Knee Surg 30: 509-513, 2017.

30. Lv TR, Q C and X. L: Treatment of posterolateral depression fractures of tibial plateau with improved postemlateral approach and $2.4 \mathrm{~mm}$ distal radiuslocking plate. Chin J Orthop Trauma 18: 851-856, 2016.

31. G F, ZJ P and H. L: Dual locking plate fixation for Type C3 tibial plateau fractures involving the posterior column. Chin J Orthop 34: 695-702, 2014.
32. Naito K, Sugiyama Y, Obata H, Mogami A, Obayashi O and Kaneko K: Screw fixation and autogenous bone graft for an irreducible distal ulna fracture associated with distal radius fracture. J Hand Surg Asian Pac Vol 22: 236-239, 2017.

33. Shi Z, Zhang X, Zhang K, Jiang R, Yang W and C. L: Short-term effect of non-absorbable sutures repairing iatrogenic medial collateral ligament injury during total knee arthroplasty. Chin J Bone Joint Surg 12: 126-130, 2019.

34. Mihalko WM, Saleh KJ, Krackow KA and Whiteside LA: Soft-tissue balancing during total knee arthroplasty in the varus knee. J Am Acad Orthop Surg 17: 766-774, 2009.

This work is licensed under a Creative Commons Attribution-NonCommercial-NoDerivatives 4.0 International (CC BY-NC-ND 4.0) License. 